\title{
Comparative Concepts for ATLAST Optical Designs
}

\author{
Bert A. Pasquale*a ${ }^{*}$, Philip Stahl ${ }^{b}$, Lee Feinberg $^{\mathrm{a}}$, Joseph Howard ${ }^{\mathrm{a}}$, Qian Gong ${ }^{\mathrm{a}}$, David Aronstein $^{\mathrm{a}}$ \\ aNASA/Goddard Space Flight Center, Greenbelt, MD 20771; \\ bASA Marshall Space Flight Center, Huntsville, AL 35812
}

\begin{abstract}
The ATALST (Advanced Technology for Large Aperture Space Telescopes) effort has presented several design incarnations. Here we will compare the design and performance of the $9.2 \mathrm{~m}$ segmented, the $8 \mathrm{~m}$ monolithic on-axis and $8 \mathrm{~m} \times 6 \mathrm{~m}$ off-axis concepts.
\end{abstract}

Keywords: Space Telescope, Large Aperture, Segmented Mirror, Monolithic, Off-Axis Design, Optical Design

\section{INTRODUCTION}

The current ATLAST design effort covers many variants, which have been reported on in response to NRC ASTRO2010 Survey $^{1}$, and have been discussed in numerous publications the past few years ${ }^{2}$. Three such designs, the $9.2 \mathrm{~m}$ segmented, the $8 \mathrm{~m}$ monolithic on-axis and the $8 \mathrm{~m} \times 6 \mathrm{~m}$ monolithic off-axis concepts are the subjects of this review. Each design shows unique characteristics and design drivers. The performance of each system is also unique and imposes limitations back on the system. This paper will discuss the design process and trades associated with each system, and compare the details and performance of each.

\subsection{Scope of Study}

ATLAST is not a single design or project - it's a general concept for an observatory which NASA and the scientific community want to build to answer what the science community asks "next." ATLAST stands for "Advanced Technology for Large Aperture Space Telescopes." This paper presents the work done as part of studies at Goddard Space Flight Center in 2008 and 2009 to examine and compare possible design options. The three designs we looked at are: $9.2 \mathrm{~m}$ Segmented, the $8 \mathrm{~m}$ Monolithic on-axis and the $8 \mathrm{~m} \mathrm{x} 6 \mathrm{~m}$ Monolithic off-axis. We will examine each of these.

\subsection{TMA Design Characteristics}

This paper will discuss the design process as a preamble to the talks about the science and use of the observatory. There is one unwavering aspect to all ATLAST designs, and that is that they are all based on the Three Mirror Anastigmat, or TMA optical design. This is simply because TMA telescopes have the highly desirable ability to produce large flat fields. (Though often a TMA design will alternately be optimized to a desired curved surface, such as a sphere centered on the exit pupil.) The general TMA design consists of a Primary, Secondary and Tertiary mirror. In Figure 1, a TMA configuration is shown with a Pupil Mirror, such as is the configuration in JWST. The typical TMA field is biased offcenter to avoid interference of the pupil and/or image with the optical beam. The TMA layout can be further modified by the use of fold mirrors, notably a field pick-off mirror located near the intermediate focus. (See Figure $1 b$.)
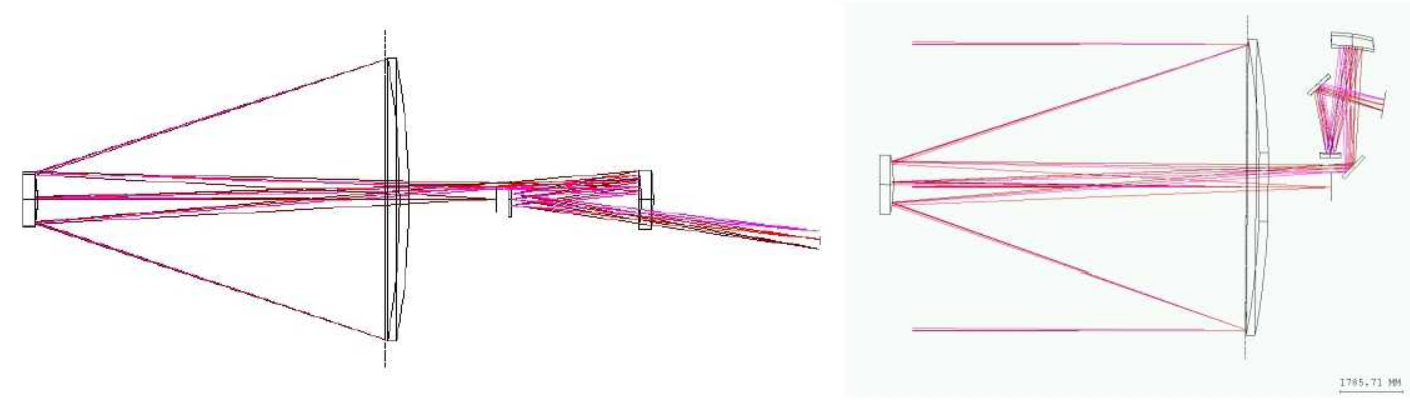

Figure 1a/b. Three Mirror Anastigmat (TMA) optical design with pupil mirror, unfolded and folded. 


\subsection{Optical Design Process}

TMA designs developed for small- to mid-class instruments do not directly translate to 8 meter UV observatories, even when other parameters such as FOV remain constant. As the instrument scales up, so do the wavefront errors. Tolerance demands also increase, and additional attention is required to meet performance specifications. A set of optimization constraints and merit functions were developed to achieve the desired results. This process involved creating a symmetric system and optimizing field performance radially in a field swath covering the science instrument fields. The weighting on various fields was then balanced iteratively.

The second thing in common with each of the designs is the departure from a "pure" TMA optical design, in that the conic of the Primary Mirror is modified to allow the intermediate focus to have an improved wavefront over a narrow field on the optical axis (suitable for specific UV instruments.) This divergence is then corrected by applying conic terms to the pupil mirror, restoring the TMA field performance. By itself, the conic on the primary mirror may also improve the TMA base performance.

\subsection{Fields of View and the TMA design}

The FOV proportions and location of the science fields directly influences the TMA design. In the example layout shown in Figure 2, the entre FOV is shown to be 8 arcminutes by 25 arcminutes, but the science fields are in the center and lower regions.

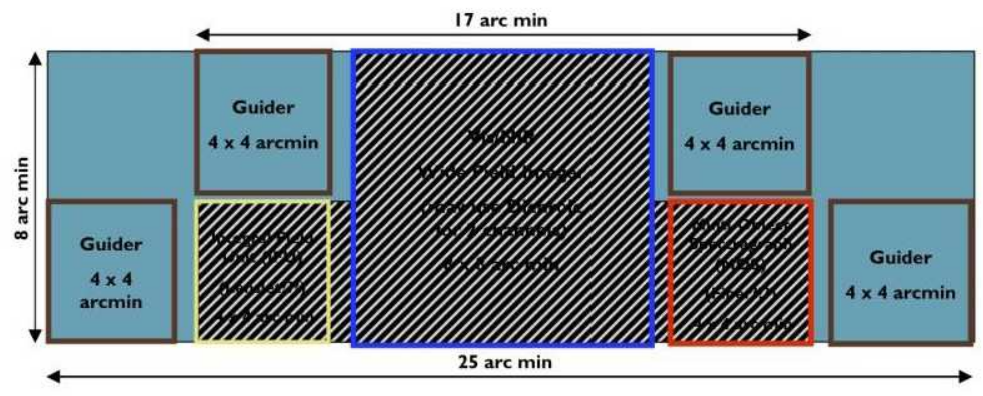

Figure 2. Sample ATLAST Field of View alocation.

Knowing that the main science instruments are located in the center and lower wings of the allocated FOV allows the designer to place the best part of the field along an arc intersecting these higher-requirement instrument fields. The outer fields may be used for guider pickoffs, which require less stringent wavefront performance and can be allowed to have a higher design residual. By offsetting the field of regard, the FOV takes on the proportions of a rectangular field. As a consequence, the best trade off between wide-field coverage and performance is often found in optimizing a field swath which is farther off the optical axis. The physical design limitation is usually assuring there is no interference between the beam and primary mirror.

\section{8 METER MONOLITHIC MIRROR DESIGN}

\subsection{Design \& Layout}

The 8-meter design was conceived as a payload for the inaugural flight of a heavy launch vehicle such as the AresV, capable of accommodating a 30 -foot diameter payload shroud. The $8 \mathrm{~m}$ monolithic primary mirror design utilizes a f/1.5 primary mirror; a solid glass substrate supported by a mounting truss behind the mirror.

The TMA in this design (and in each of the three we are presenting for consistency in comparison) has an f/18 flat focal plane. Because of the symmetric space behind the primary mirror provided by a heavy launch vehicle, dual TMA channels can be implemented on opposite sides of the optical axis, as shown in Figure 3a. The 8m TMA optical layout was designed with compact back ends that can be folded up behind the primary mirror and simultaneously have an accessible Cassegrain Focus. The tertiary mirrors are then embedded in, and supported by, the primary mirror support structure. As long as the optical beams do not interfere with main support rings and trusses, this is quite feasible. Additional fold mirrors allow the final TMA focal plane to be relayed to the center instrument space, or the instrument can also be integrated behind the primary mirror as shown in Figure $3 \mathrm{~b}$. 

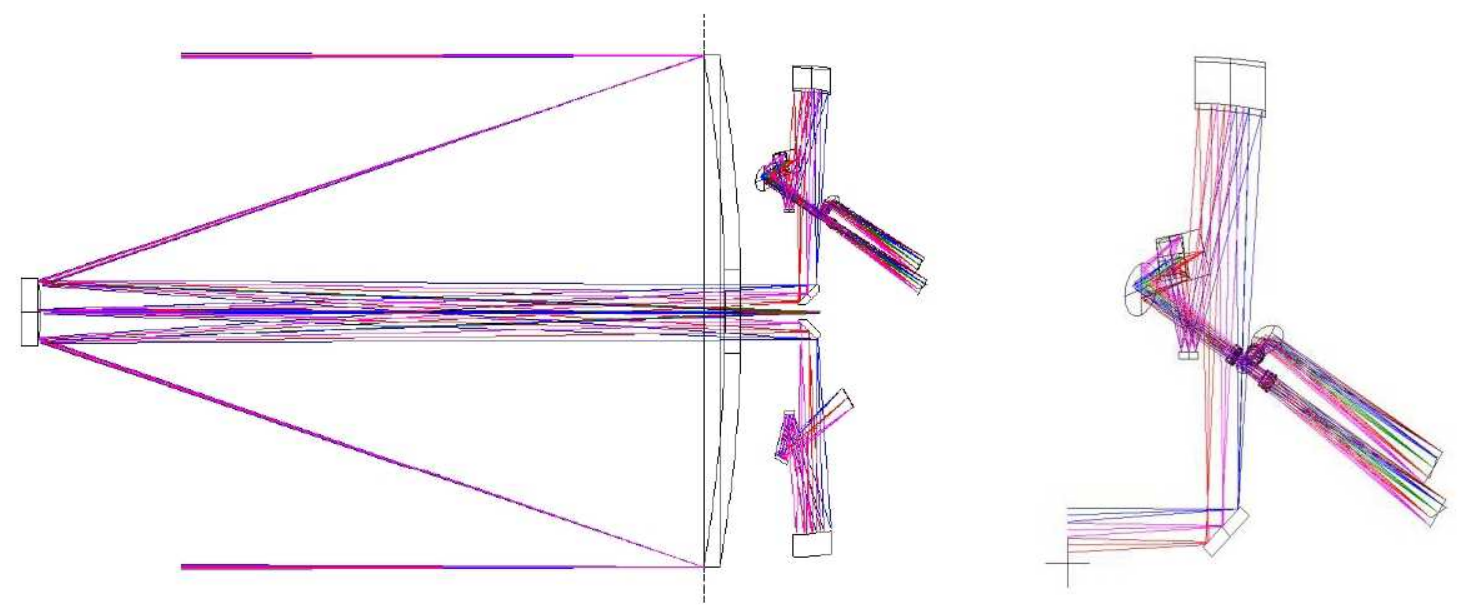

Figure $3 \mathrm{a} / \mathrm{b}$. The $8 \mathrm{~m}$ monolith optical layout with dual TMA fields shown, and a closeup of a Visible/IR Wide-Field concept.

The Primary/Secondary combination produces an intermediate "Cassegrain focus" with an $f / 13.63$ convergence. This is where the UV instruments may access an image that has had only two reflections and has maintained high UV throughput. The precise qualities of the Cassegrain image can be tailored to the instruments finally slated for implementation.

\subsection{Fields of View}

The location of the TMA fields is constrained only by the physical limits of the optical system. Figure 4 shows this particular TMA dual fields' inner edge at either 4 or 5 arcminutes from the Optical Axis. As before, the particulars of the optical design can be made to accommodate the necessary fields for the particular science instrument compliment.

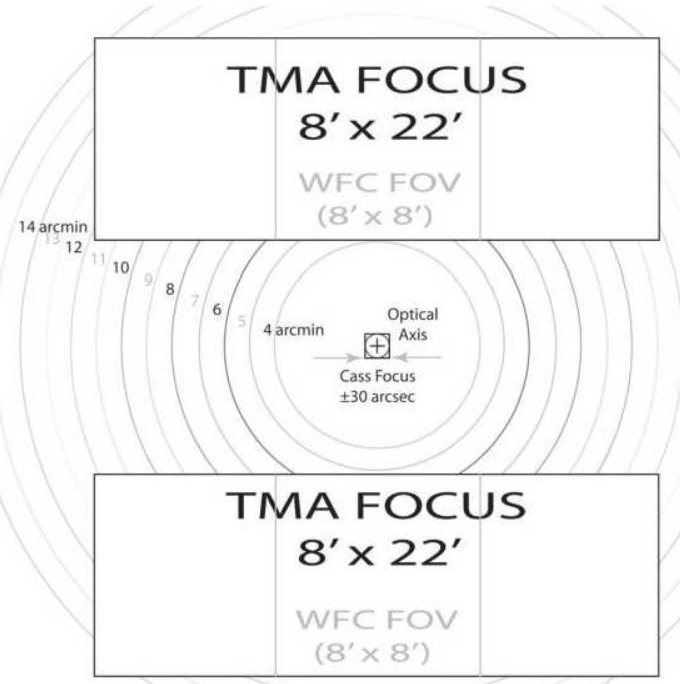

Figure 4. The center UV Cassegrain field and dual TMA fields shown together.

\subsection{TMA Performance}

During the design process, we first optimize the TMA fields so that the sweet spot of the wavefront performance will cover the wide field camera FOV, allocated in this design within the swath 4 arcminutes to 13 arcminutes off axis. The Primary Mirror conic is then allowed to deviate from the standard TMA design parameters to improve the UV focus region, and finally aspheric terms are added to the pupil mirror to restore the TMA Field performance. Figure 5 shows the resulting wavefront, shown in nm RMS, across the radial field. 


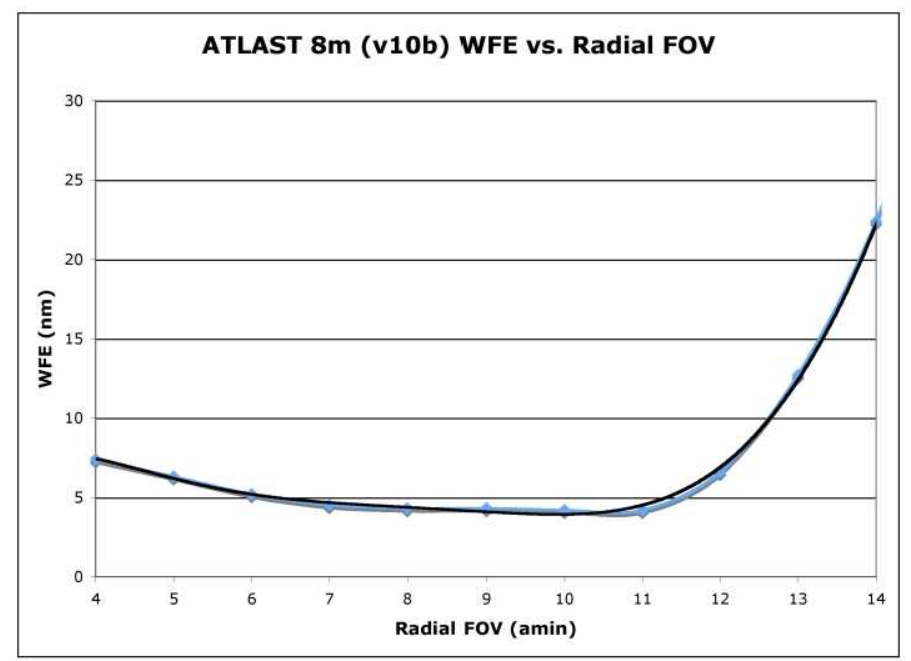

Figure 5. The 8m TMA wavefont RMS (nm) as a function of off-axis angle.

From 4 to 12.5 arcminutes off axis, the wavefront is less than $10 \mathrm{~nm}$ RMS, and from 5 to 12 arcminutes it's less than 6 $\mathrm{nm}$ RMS. When you take that curve and sweep it radially across the entre TMA FOV, you can see how much field is ideal for science instruments. The entre $8 \times 8$ arcminute Wide Field Camera FOV has performance 4-10 nm RMS, with $75 \%$ of the field is $<5 \mathrm{~nm}$ RMS.

Figure 6a shows this curve swept across the full $8 \times 20$ arcminute field, and Figure $6 \mathrm{~b}$ a 3-D projection of the wavefront map across the same field. Guiders or low spatial resolution instruments could be placed in the far wings where RMS requirements can be relaxed.
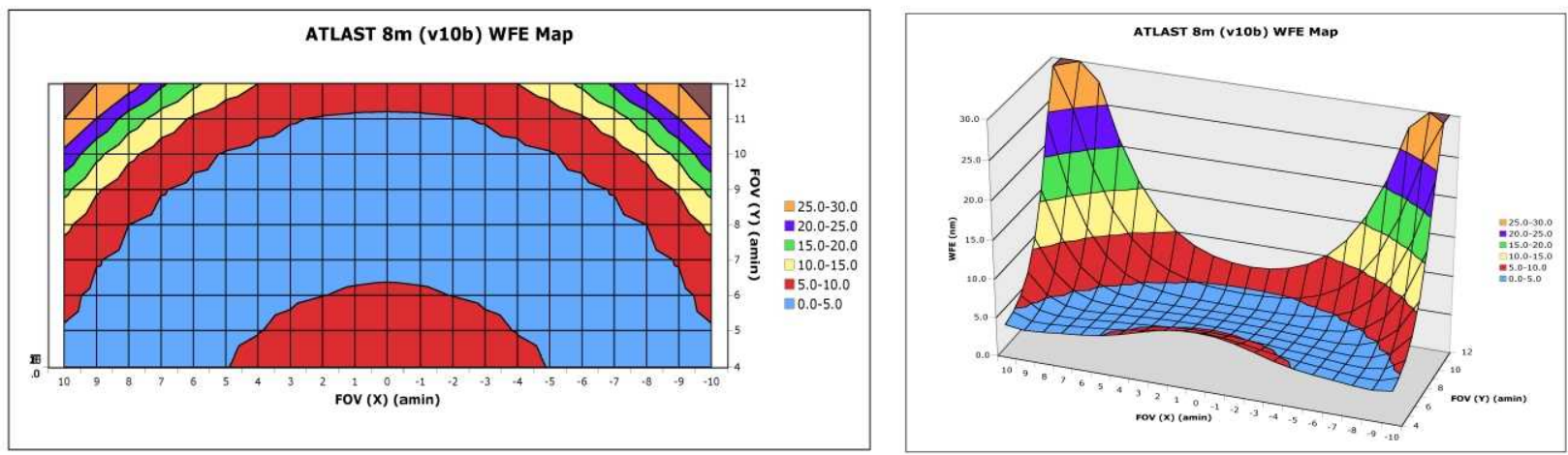

Figure $6 \mathrm{a} / \mathrm{b}$. The $8 \mathrm{~m}$ wavefront quality over the TMA field.

\subsection{UV Cassegrain Performance}

If we peal away the back end of the TMA (Figure 7a) and just focus on the Cassegrain section we can see where the UV science instruments can be placed. This is a very narrow field, but sufficient for many spectrometers or coronagraph type of instrumentation. As you can see in Figure 7b, even with the modified design, the wavefront increases almost linearly as you move away from the optical axis. There is a 10 arcsecond field where the RMS is less than $10 \mathrm{~nm}$, and a 20 arcsecond field where the RMS is less than $20 \mathrm{~nm}$. The primary and secondary mirrors would have been treated with high-reflectance UV coatings, but at the expense of decreased throughput in the visible and IR. Further correction could be done within the UV instrument to allow increased field use, but you would still want to minimize the number of reflections. 

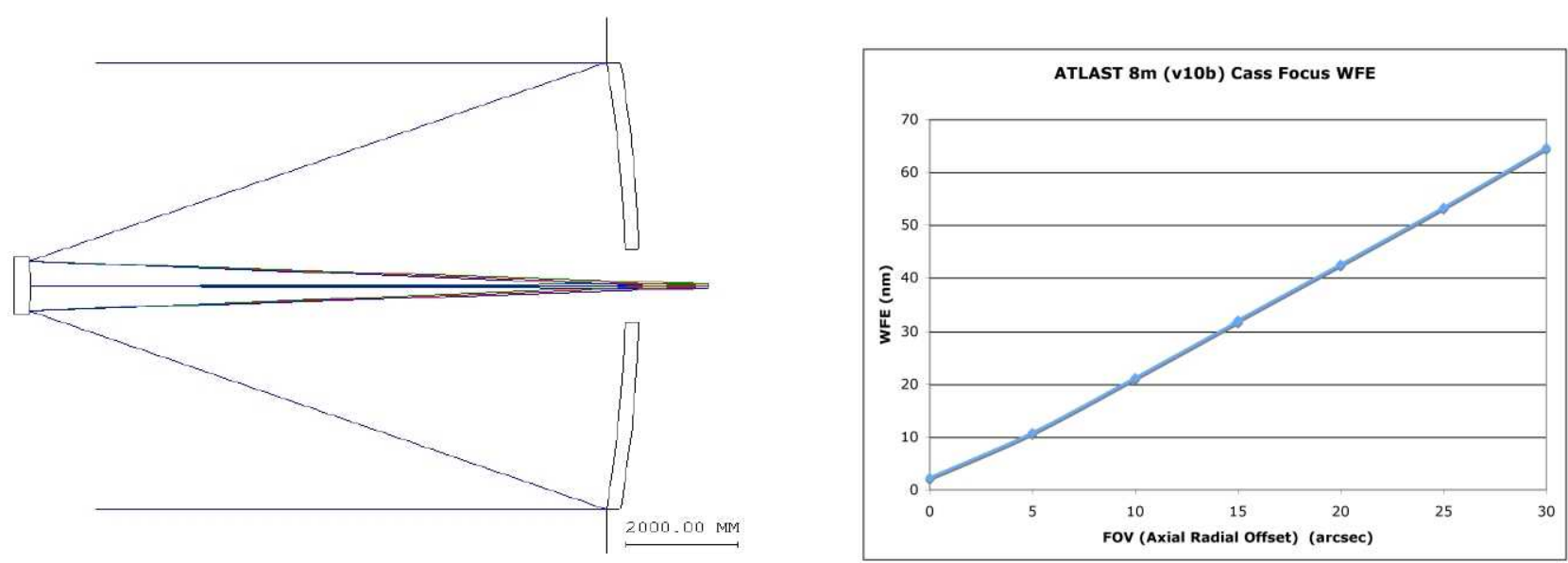

Figure $7 \mathrm{a} / \mathrm{b}$. The "Cassegrain" telescope section, and the wavefront error as a function of field.

\subsection{Error Budget Constraints}

To understand why the emphasis on wavefront performance is so tight, we must look at the overall budget for the observatory. This is shown in Table 1:

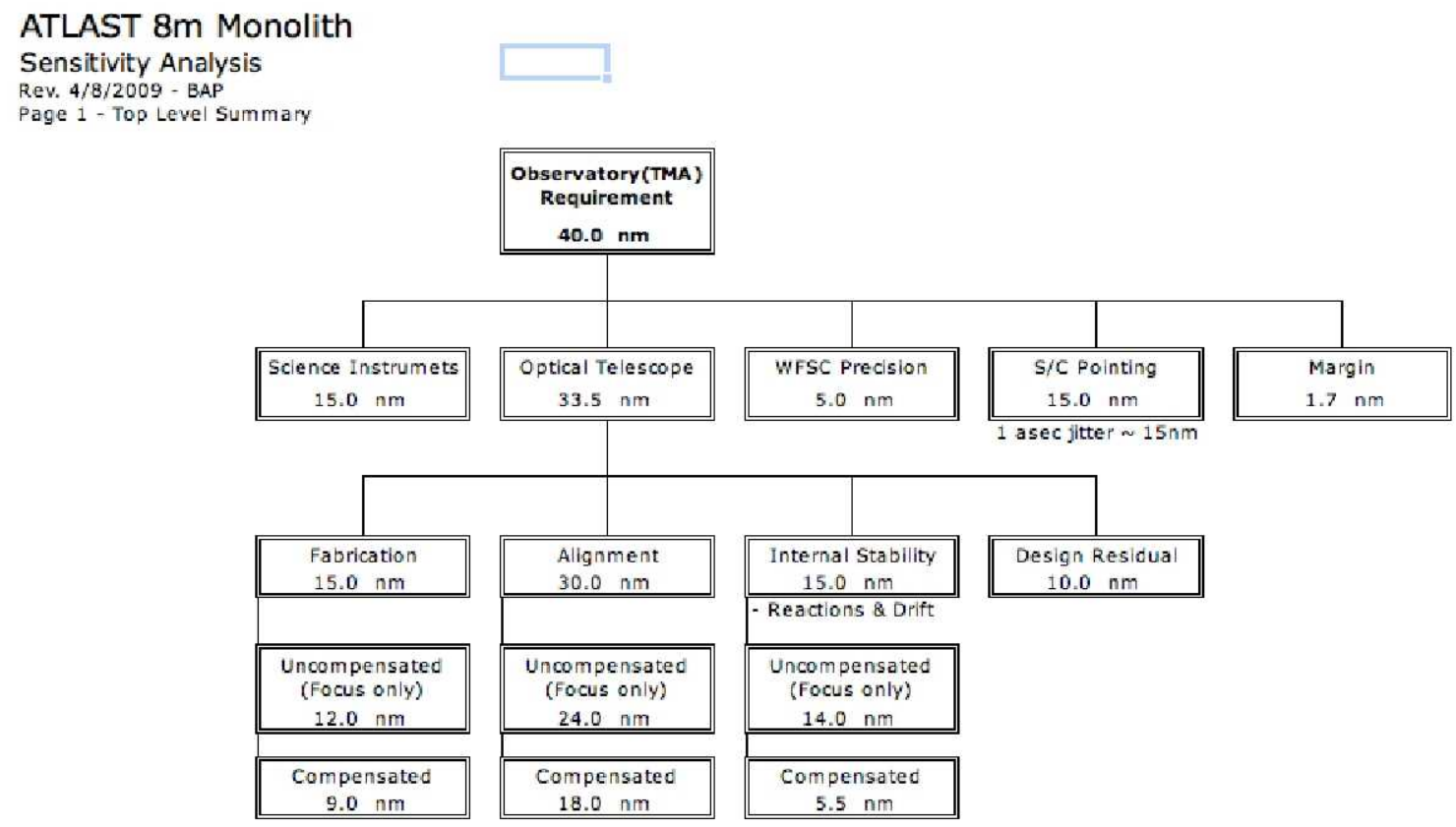

Table 1. Error Budget for the ATLAST concept, allocating 10nm RMS to the TMA Design.

The entire RMS wavefront budget for imaging in the observatory is only $40 \mathrm{~nm}$ ! In this chart you see Telescope "Design Residual" is only allocated 10nm. This is RSSed with all the fabrication, alignment and stability error sources. So any design error over $10 \mathrm{~nm}$ will burden the budget, any less provides margin to the other parameters. 


\section{9.2 METER SEGMENTED MIRROR DESIGN}

\subsection{Design \& Layout}

The second design looked at was the 9.2 segmented concept, which was referred to as ST-2020 during the study. This study is the "non-ultra heavy lift vehicle" option, designed to be accommodated on a heavy-up Delta rocket. The segments are identical in profile to JWST $-1.315 \mathrm{~m}$ across, flat to flat. By adding the third ring of 18 segments, the total entrance aperture diameter becomes $9.2 \mathrm{~m}$, and literally twice the collecting area as JWST. Like JWST the outer segments would fold into the narrow shroud. (See Figure $8 \mathrm{a} / \mathrm{b}$. $9.2 \mathrm{~m}$ design configuration, a) stowed for launch and $\mathrm{b}$ ) deployed., below.)
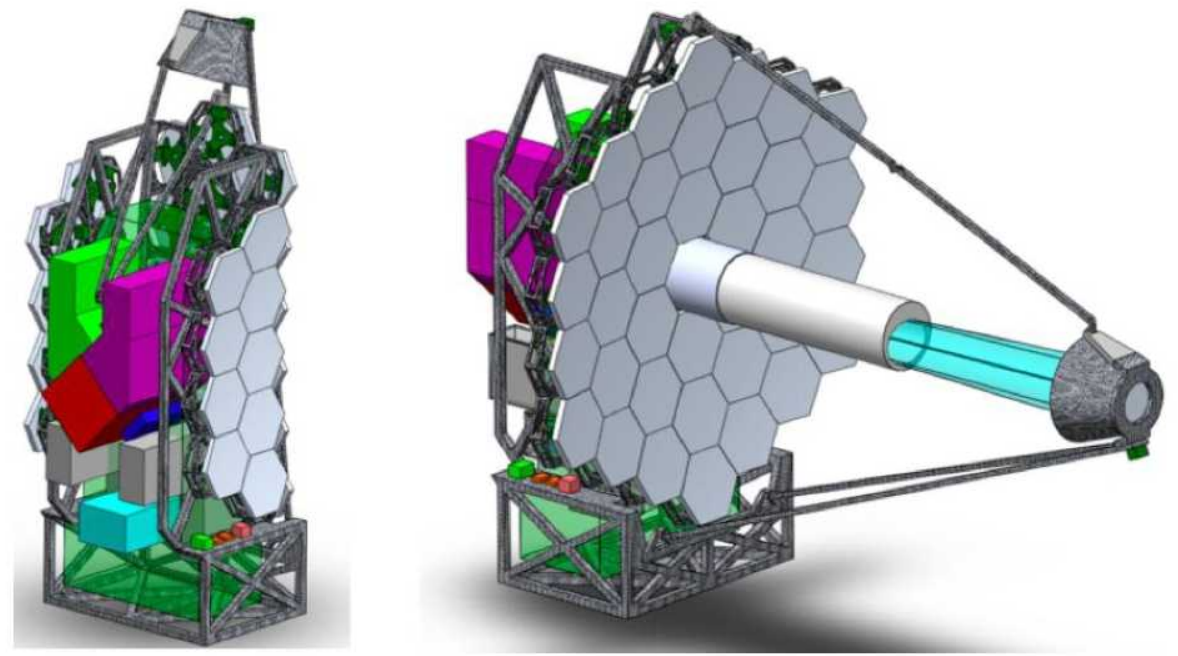

Figure $8 \mathrm{a} / \mathrm{b}$. 9.2m design configuration, a) stowed for launch and b) deployed.

In this design, the optical parameters are very similar to the $8 \mathrm{~m}$ design, though the primary mirror's power had to be increased to $\mathrm{f} / 1.25$ (vs. $\mathrm{f} / 1.5$ for the $8 \mathrm{~m}$ monolith.) The internal focus shifted correspondingly to $\mathrm{f} / 12.5$, but the TMA was maintained at $\mathrm{f} / 18$. It is again a modified TMA w/ improved Cassegrain focus for UV instruments, and the back end is designed for a compact folding in the aft space. 


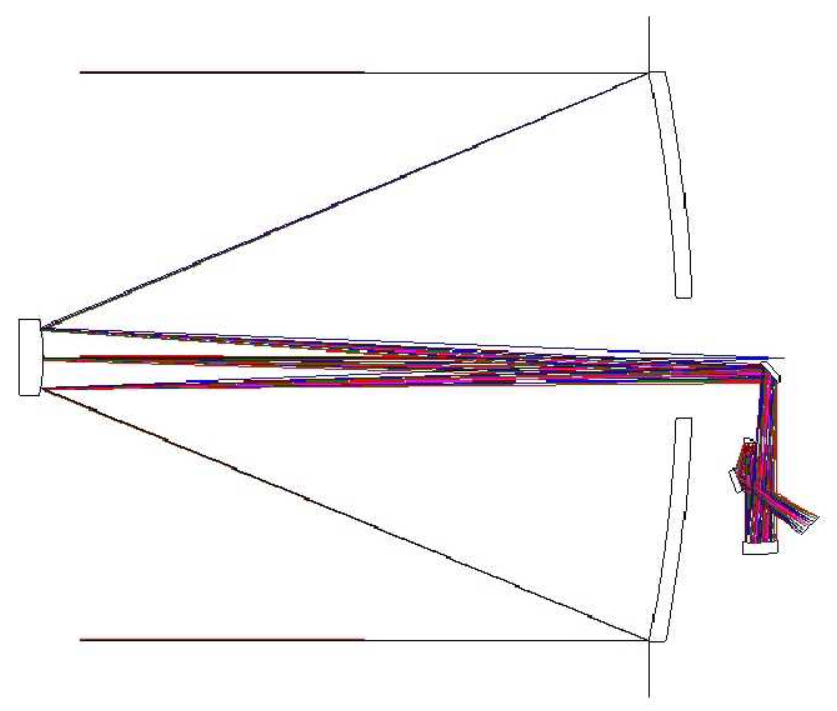

$2000.00 \mathrm{Mm}$

Figure 9. The 9.2m Segmented telescope optical layout.

This is the basic layout of the 9.2m TMA, with the UV focus accessible and the TMA Focal plane biased toward the bottom of the shroud where the instrument bay is located. The actual focal plane position can be placed as desired as the instrument compliment is defined.

\subsection{Fields and TMA Performance}

The TMA field was configured to have its inner edge 4 arcminutes from the optical axis. The initial design used an 8 " 20 " field, but the central hole in the primary mirror could accommodate a 23 arcminute wide field. The FOV layout, and the wavefront performance across the swath of field covering the science area are shown in Figure $10 \mathrm{a} / \mathrm{b}$.
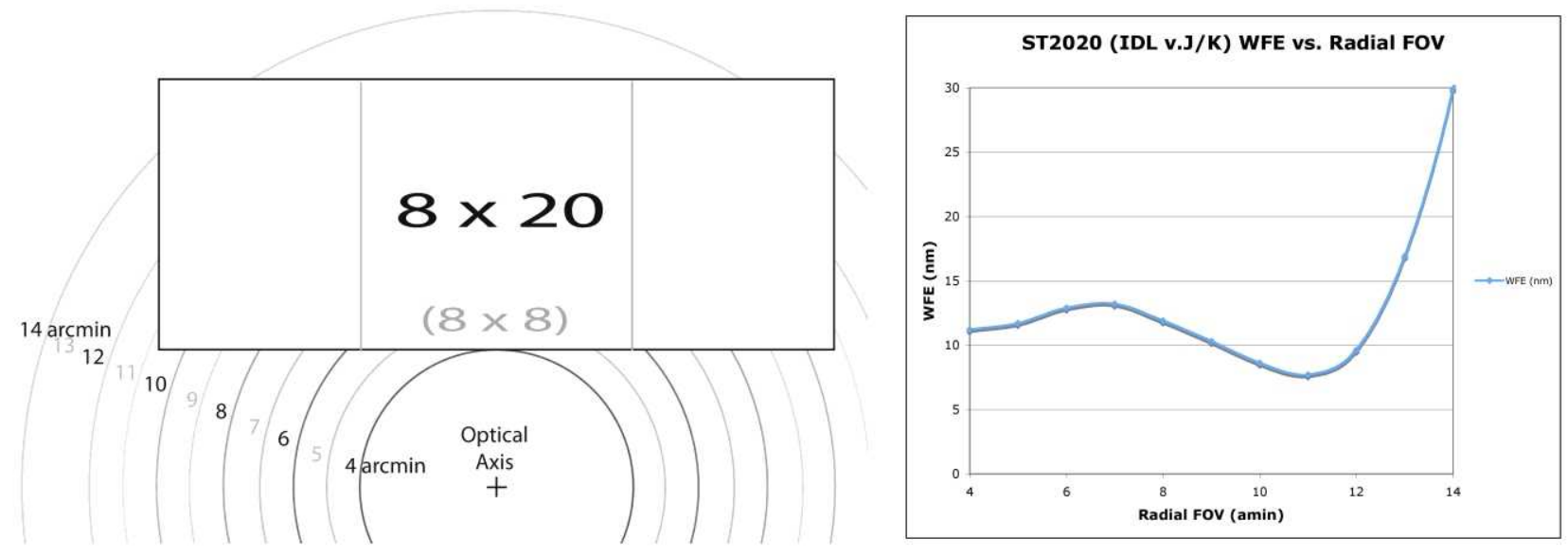

Figure $10 \mathrm{a} / \mathrm{b}$. The $9.2 \mathrm{~m}$ field allocation and wavefront performance.

The field performance is mostly comparable to the previous design - under $12 \mathrm{~nm}$ from 4 to 12.5 arcminutes. The main contributor for it being 1-5 nm greater than the $8 \mathrm{~m}$ monolithic design is the faster Primary Mirror. However, performance is still excellent in a wide area, as shown in the Figure $11 \mathrm{a} / \mathrm{b}$. 

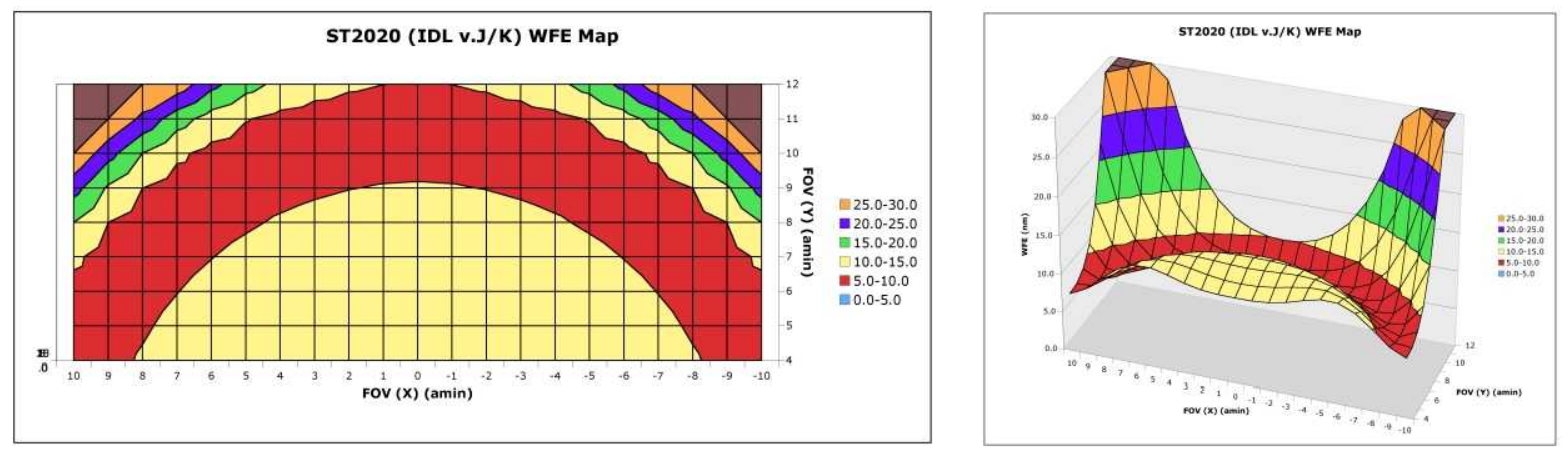

Figure $11 \mathrm{a} / \mathrm{b}$. The $9.2 \mathrm{~m}$ telescope field performance and 3-D projection.

\subsection{UV Cassegrain Performance}

Like the $8 \mathrm{~m}$ design, the $9.2 \mathrm{~m}$ system has a narrow field of focus at the intermediate focus, useable by various UV instruments. The faster primary mirror and Cassegrain focus constrain the size of high-quality field achievable. The field for RMS $<10 \mathrm{~nm}$ is 8 arcseconds, and $<20 \mathrm{~nm}$ is 16 arcseconds. (See Figure 12a/b.) Again, the UV instruments can incorporate some level of correction into their design, but reflections must me minimized to maintain UV throughput.
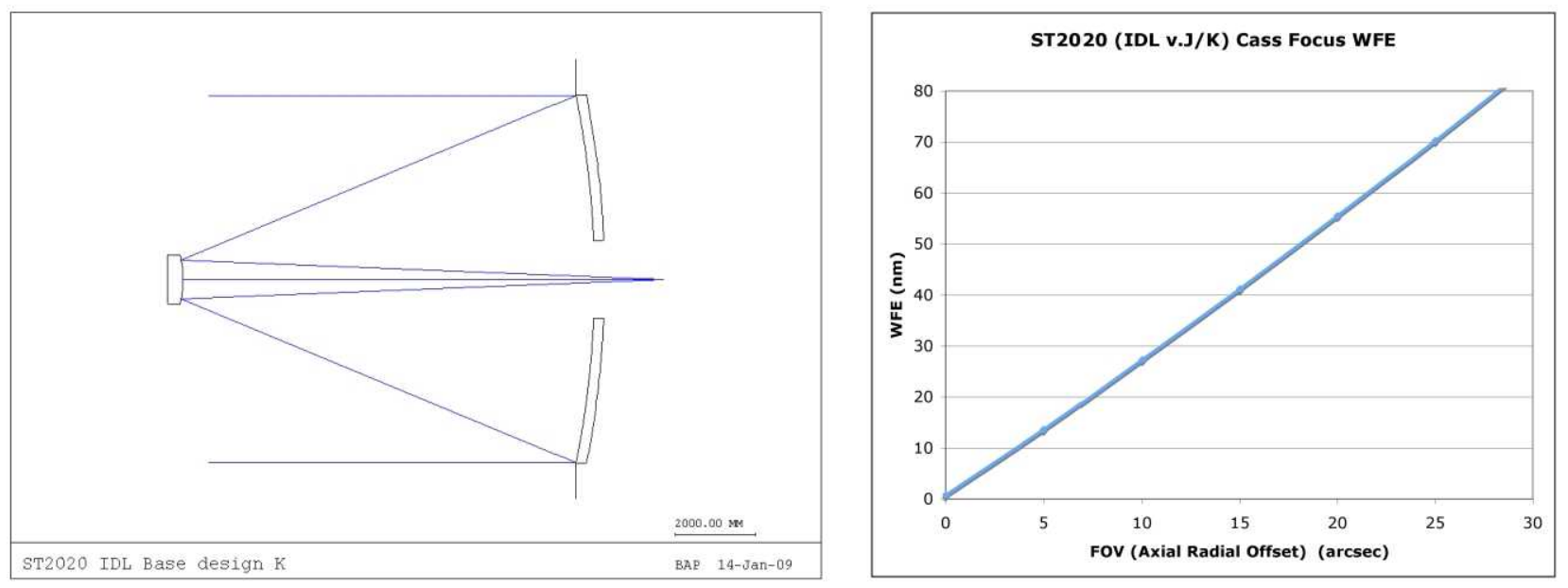

Figure $12 \mathrm{a} / \mathrm{b}$. The $9.2 \mathrm{~m}$ telescope intermediate focus and performance.

\section{6 X 8 METER OFF-AXIS MIRROR DESIGN}

\subsection{Design \& Layout}

While the first two designs compared on-axis TMA designs, the final configuration or discussion is the $6 \mathrm{~m} \times 8 \mathrm{~m}$ monolithic Off-Axis TMA design. This is further related to the $8 \mathrm{~m}$ monolith design in that it is also envisioned for a heavy launch vehicle with a $30 \mathrm{~m}$ payload shroud, as shown in Figure 13 . Everything is comparable to the general aspects of the on-axis designs, except that the Primary Mirror had to be slowed down to $f / 1.8$ since the system is in essence using a section of mirror wider than even the $9.2 \mathrm{~m}$ design - the entire parent mirror would be 14 meters in diameter. The TMA maintains $\mathrm{f} / 18$ focus (flat plane, orthogonal to Optical Axis) but the Cassegrain focus slows slightly to $\mathrm{f} / 15$. The TMA back-end is folded behind the primary mirror, making both the Cassegrain focus and TMA focal plane accessible. Can accommodate extra-large pickoff field (in lieu of using Cass Focus.) Though shown here with a single pick-off mirror, 

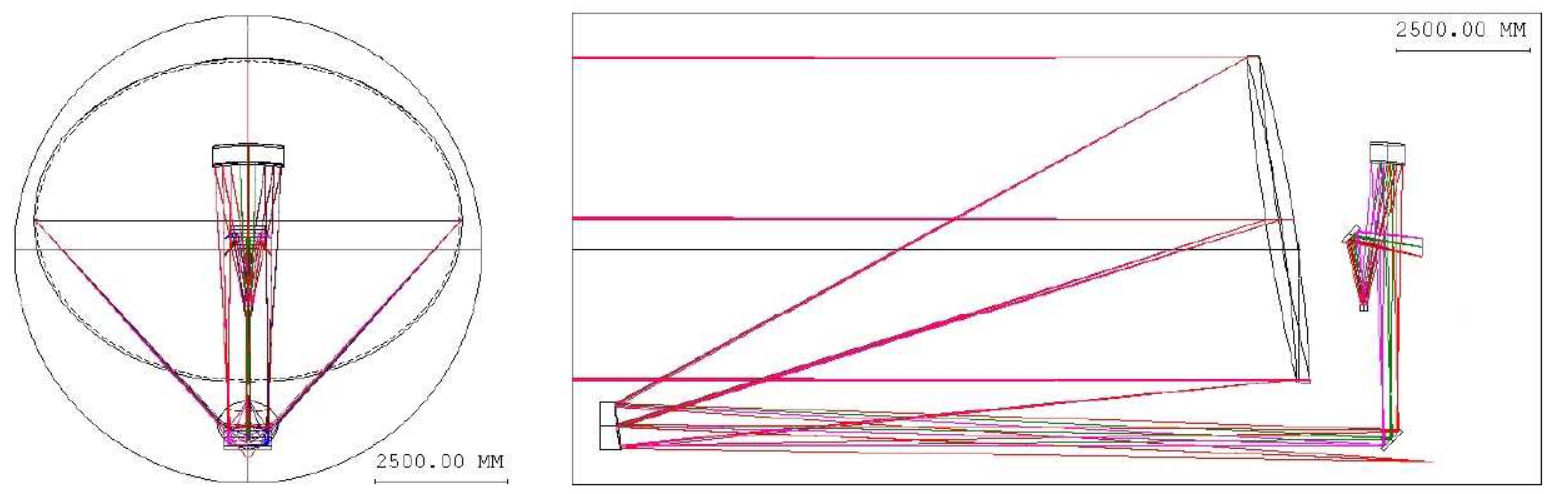

Figure 13. Off-Axis 6x8 meter TMA shown in a 30-foot diameter shroud.

\subsection{Fields \& Performance}

The off-axis design's FOV is also more skewed away from the optical axis than the on-axis designs. (See Figure 14.) The FOV of interest begins about 7 arcminutes from the axis, and continues out to 16 or 17 arcminutes. So, we needed to design this with a very different wavefront profile. And here you see that from 7 to 16.5 arcminutes are below $10 \mathrm{~nm}$ RMS, while the entire range from 8 to 15 is below $5 \mathrm{~nm}$ RMS.
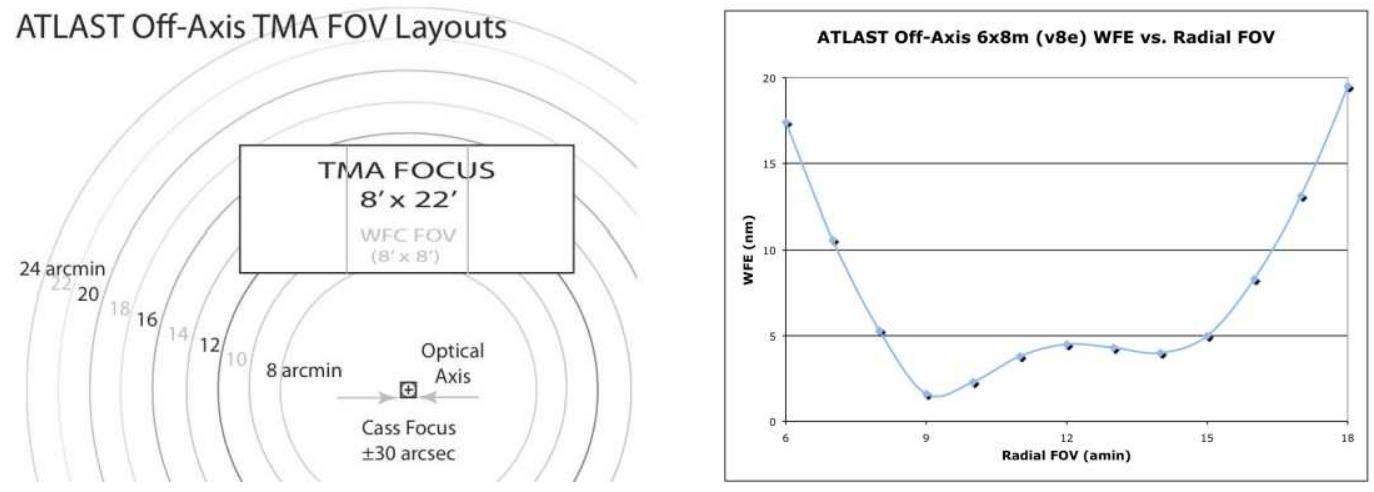

Figure 14. Off-Axis Field and wavefront performance.

Again a large TMA FOV is able to accommodate various science instruments, as shown in Figures 15:
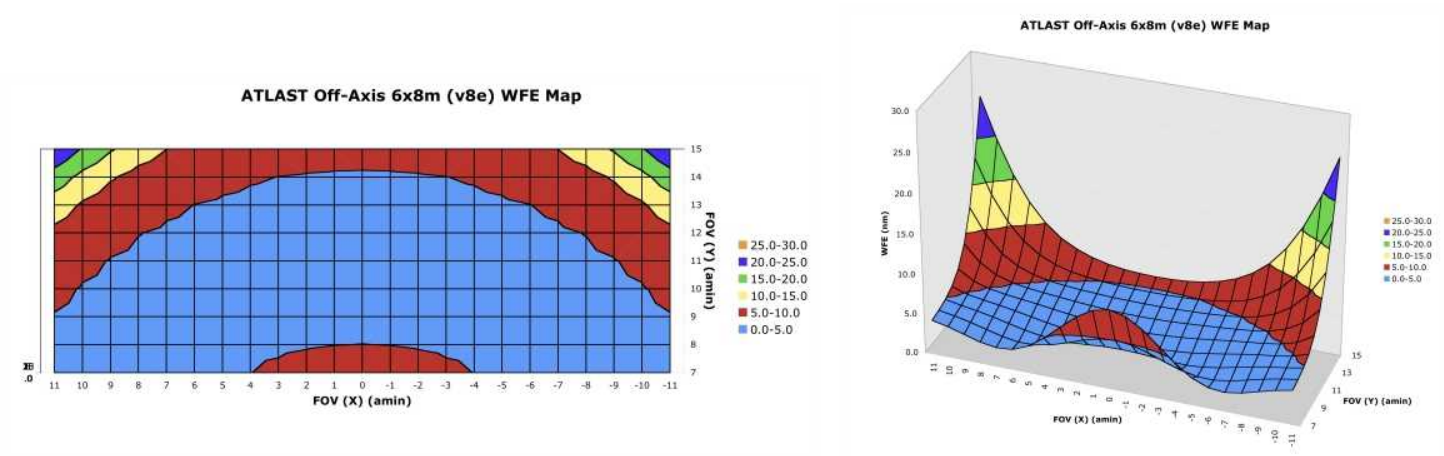

Figures 15a/b. 6m x 8m Wavefront RMS (nm) over the TMA Field of View.

\subsection{Expanded Field Options}

The Off-axis TMA design can actually accommodate an expanded FOV if desired. This provides plenty of prime real estate for additional science instruments, as shown in Figure 16. Another option is to allow instruments to pick off fields 
to either side of the optical axis, though additional folds and tertiary mirrors would have to be integrated into their design.
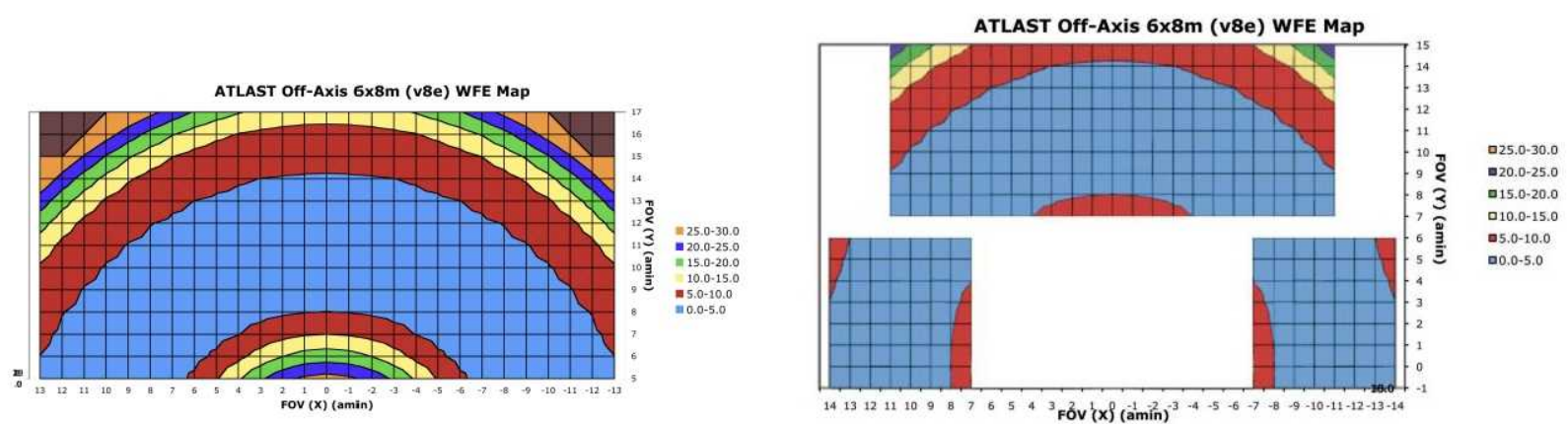

Figure 16. Options for an expanded FOV for the Off-Axis Monolith Design.

Unlike the on-axis designs, the $6 \mathrm{~m} \times 8 \mathrm{~m}$ off-axis design does not afford a well-corrected intermediate focus using the method described. This seems to be a limitation of using such an off axis section of the parent pupil. Instruments placed at the Cassegrain focus would have to have internal correction built in. Ideally, the telescope design would be reworked in tandem with instrument designs to maximize performance.

\section{COMPARISION \& SUMMARY}

\subsection{Component Sizes}

Tables 2-4 show the sizes of the optical components for the 8m Monolith, 9.2m Segmented, and 6x8m Off-Axis designs.

\begin{tabular}{|c|c|c|c|c|}
\hline & Radius (mm) & Conic & 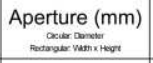 & Other \\
\hline Primary & $24,000.00$ & -0.994680 & C 8,000 & Concave \\
\hline Secondary & 3006.791 & -1.487700 & C 1,050 & Convex \\
\hline Fold 1 & Flat & 0 & R $780 \times 480$ & \\
\hline Tertiary & $3,414.393$ & -0.729426 & $\begin{array}{l}R 1,150 \times 590 \\
350 \text { Off-Axis }\end{array}$ & Concave \\
\hline Pupil & Flat & $\begin{array}{l}\text { 4th-order Aspheric: } \\
-0.292698 \mathrm{e}-10 \mathrm{X} \\
-0.205033 \mathrm{e}-10 \mathrm{Y}\end{array}$ & C 160 & $\begin{array}{c}\text { (Flat" with } \\
\text { anamorphic } \\
\text { aspheric terms) }\end{array}$ \\
\hline Fold 2 & Flat & 0 & R $620 \times 220$ & \\
\hline
\end{tabular}

Table 2. $8 \mathrm{~m}$ monolithic design optical component sizes.

\begin{tabular}{|l|c|c|c|c|}
\hline & Radius $(\mathrm{mm})$ & Conic & $\begin{array}{c}\text { Aperture }(\mathrm{mm}) \\
\text { ander }\end{array}$ & Other \\
\hline Primary & $23,000.000$ & -0.996326 & $\mathrm{C} 9,200$ & Concave \\
\hline Secondary & $2,665.3656$ & -1.442873 & $\mathrm{C} 1,100$ & Convex \\
\hline Fold 1 & Flat & 0 & $\mathrm{R} 760 \times 440$ & \\
\hline Tertiary & $2,942.0139$ & -0.762194 & $\begin{array}{c}\mathrm{R} 1,160 \times 560 \\
320 \text { Off-Axis }\end{array}$ & Concave \\
\hline Pupil & Flat & $\begin{array}{c}\text { A: }-0.428242 \mathrm{e}-10 \\
\text { B: } 0.108072 \mathrm{e}-14\end{array}$ & $\mathrm{C} 150$ & $\begin{array}{l}\text { (4th \& 6th order } \\
\text { aspheric terms) }\end{array}$ \\
\hline Fold 2 & Flat & 0 & $\mathrm{R} 440 \times 260$ & \\
\hline
\end{tabular}

Table 3. 9.2m segmented design optical component sizes. 


\begin{tabular}{|c|c|c|c|c|}
\hline & $\begin{array}{c}\text { Radius } \\
(\mathrm{mm})\end{array}$ & Conic & 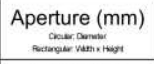 & Other \\
\hline Primary & $28,800.00$ & -0.991702 & $\begin{array}{c}E 8,000 x \\
6,000\end{array}$ & Concave \\
\hline Secondary & $4,117.562$ & -1.526240 & $\begin{array}{c}\text { E } 1,140 \times 880 \\
600 \text { Off-Axis }\end{array}$ & Convex \\
\hline Fold 1 & Flat & 0 & $R 850 \times 500$ & \\
\hline Tertiary & $4,717.870$ & -0.695919 & $\begin{array}{c}R 1,200 \times 620 \\
360 \text { Off-Axis }\end{array}$ & Concave \\
\hline Pupil & Flat & $\begin{array}{l}\text { 4th-order Aspheric: } \\
0.312237 \mathrm{e}-16\end{array}$ & C 160 & $\begin{array}{l}\text { ("Flat" with } \\
\text { aspheric terms) }\end{array}$ \\
\hline Fold 2 & Flat & 0 & $\mathrm{R} 620 \times 220$ & \\
\hline
\end{tabular}

Table 4. $6 \mathrm{~m} \times 8 \mathrm{~m}$ off-axis design optical component sizes.

\subsection{Primary $\mathbf{f} / \#$ and TMA Performance}

One very interesting point that came from this study is the relationship of Primary $f / \#$ to best wavefront performance. Because in each design a wide radial field ( $\sim 10$ arcminutes) was being maintained in optimization, the average wavefront error was inversely related to the Primary Mirror f/\#.

The monolithic designs take advantage of the heavy lift vehicle capacity to avoid phasing segments. Only the off-axis monolith provides an unobstructed pupil, desirable for $\mathrm{e}^{-10}$ coronagraphy of exoplanets.

\section{REFERENCES}

[1] Marc Postman, et.al., "Advanced Technology Large-Aperture Space Telescope (ATLAST): A Technology Roadmap For The Next Decade,” R.F.I. Submitted to NRC ASTRO-2010 Survey (2009).

[2] http://www.stsci.edu/institute/atlast/index html_documents page 